\title{
Gastric Schwannomas: Rare Gastric Mesenchymal Tumor
}

\author{
Anuradha Sekaran', Sandeep Lakhtakia ${ }^{2}$, Darisetty Santosh ${ }^{3}$, Rajesh Gupta ${ }^{2}$, Guduru Venkat Rao ${ }^{4}$, \\ Duvvuru Nageshwar Reddy ${ }^{2}$
}

Departments of ${ }^{1}$ Pathology, ${ }^{2}$ Medical Gastroenterology, ${ }^{3}$ Anaesthsiology and ${ }^{4}$ Surgical Gastroenterology, Asian Institute of Gastroenterology, Hyderabad, India

\section{Introduction}

Gastric schwannomas are uncommon benign mesenchymal tumors of the gastrointestinal tract. Clinically they are often included in the broad group of stromal tumors. However they have a characteristic histology that differentiates them from stromal tumors. Microscopically, spindle cell

\section{Case 1}

A 40-year-old female presented with upper abdominal discomfort and dyspepsia. She had hypothyroidism for five years on regular hormone replacement treatment. Clinical examination was non-contributory.

Ultrasound abdomen showed a hypoechoic mass in the

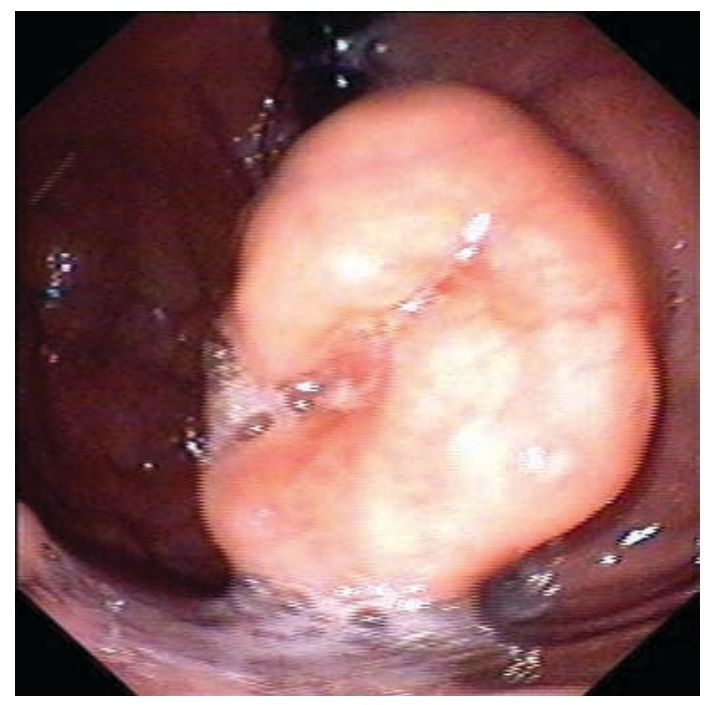

Figure 1(a): Polypoidal mass in body of stomach.

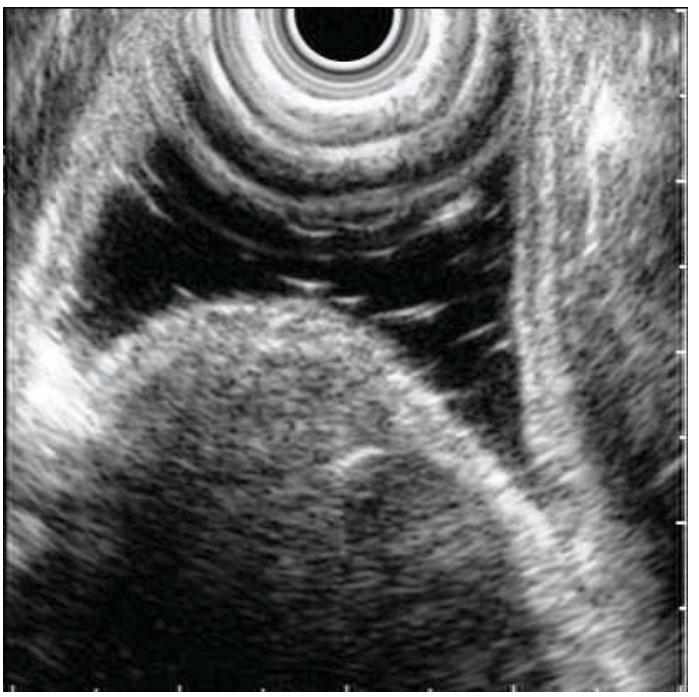

Figure 1(b): Hyperechoic mass arising from muscularis propria tumors need to be interpreted with immuno-histochemical markers to accurately differentiate schwannomas from other mesenchymal tumors. Gastrointestinal schwannomas have an excellent prognosis after surgical resection.

We report here, three cases of gastric schwannomas, all of which appeared as gastric polypoidal lesion on upper gastro-intestinal endoscopy, with one case presenting with upper gastrointestinal bleeding.
Reprints requests and correspondence:

Dr. Anuradha Sekaran

Consultant Pathologist,

Asian Institute of Gastroenterology, Hyderabad - INDIA.

91-40-23378888, Ext: 713, Fax: 91-40-23324255.

Mobile: +91-9848134581

Email: sanuradha_dr@yahoo.co.in 
hepato-gastric area, which was indenting the lesser curvature of stomach. Upper gastrointestinal endoscopy (UGIE) revealed an polypoidal mass in the body of stomach along lesser curve (Figure 1a). Endoscopic ultrasonography (EUS) showed a large mass (Figure 1b) arising from muscularis propria (fourth layer) of gastric wall. CECT scan upper abdomen confirmed an extrinsic mass in the stomach suggestive of soft tissue tumor. Patient underwent laparotomy with sleeve resection of gastric tumor and pyloromyotomy.

The gross specimen consisted of polypoidal mass, measuring $4.5 \times 4.5 \times 2 \mathrm{~cm}$. Sections showed well circumscribed mass, homogenous gray to white, shiny, firm to rubbery surface with focal whorled areas. Microscopic examination of hematoxylin - eosin stained sections showed a circumscribed unencapsulated moderately cellular tumor composed of spindle shaped cells arising from submucosa and involving muscularis propria. Cells were arranged in intersecting fascicles and storiform pattern displaying mild nuclear pleomorphism, and evenly distributed chromatin. Mitotic figures were $3 / 50$ high power fields. Focal dense infiltration by lymphocytes and plasma cells along with few lymphoid follicles with germinal centers were noted which more pronounced at the periphery of tumor.

The tumor cells showed diffuse intense nuclear and cytoplasmic positivity for S-100 protein, and were non reactive for CD 117, DOG1, CD 34 and smooth muscle actin (SMA), Ki67 index was 2\%. Morphology and immunohistochemical profile confirmed gastric schwannoma.

\section{Case 2}

A 35-year-old female patient presented with recurrent epigastric and left upper abdominal pain associated with bilious vomiting for two years. On clinical evaluation patient was anemic. Hemoglobin was 9gm/dl (normal 12-15gm/dl). Biochemical and hematological profile was suggestive of iron deficiency anemia.

Ultrasound abdomen revealed cholelithiasis and a gastric antral wall hypoechoic mass lesion. UGIE showed a broad based polypoidal lesion in the gastric antrum (Figure 2). EUS showed a well defined hypo-echoic gastric antral polyp localized in the muscularis propria layer of gastric wall, suggestive of leiomyoma or gastrointestinal stromal tumor (GIST). Patient underwent laparoscopic gastric sleeve resection and cholecystectomy.

Gross specimen showed a polypoidal growth of $3 \times 3 \times$ $1 \mathrm{~cm}$. Cut section showed gray tan mass with whorled pattern having focal myxoid areas. There was no hemorrhage or necrosis. On histology a moderately cellular spindle cell tumor arising from muscularis propria was noted. Nuclear atypia was minimal. Intervening stroma showed scattered lymphocytes and plasma cells with numerous lymphoid follicles at the periphery. Mitotic figures were 2/50 high power fields. In addition focal myxoid areas, abundant collagen and areas of hyalinization were noted.

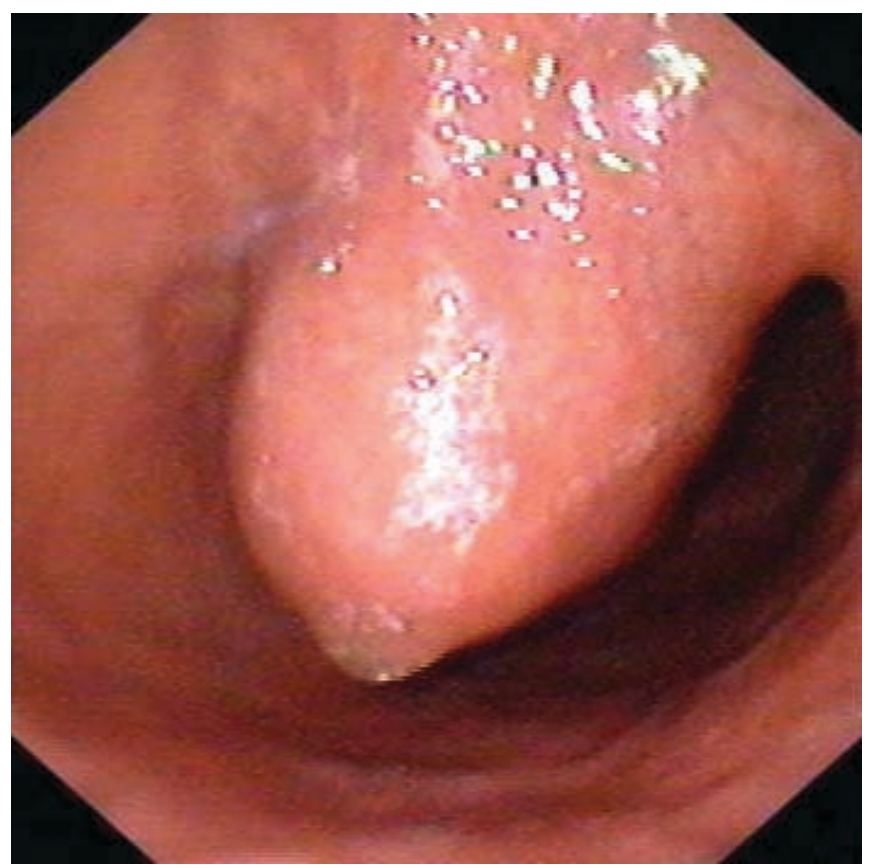

Figure 2 : Endoscopy showing broad based polypoidal lesion

IHC showed diffuse and intense nuclear and cytoplasmic positivity for S-100 protein. There was no reaction for $\mathrm{CD} 117, \mathrm{CD} 34, \mathrm{DOG} 1$ and smooth muscle actin (SMA), Ki67 index was 1\%. Based on morphology and immuno-histochemical profile final diagnosis was that of gastric schwannoma.

\section{Case 3}

A 43 year old male patient presented with symptoms of dyspepsia and altered bowels for six months. He underwent surgery twice for carcinoma rectum in last six years. Anterior resection was performed six years ago followed by chemotherapy and radiotherapy. Subsequently he underwent abdominoperineal resection (APR) for local recurrence of carcinoma rectum four years ago.

His hematological and biochemical parameters were within normal range. CEA was $8.2 \mathrm{ng} / \mathrm{ml}$ (normal < 5ng/ml). Ultrasound abdomen was normal. CT scan abdomen showed moderately enhancing soft tissue mass measuring 5.2 $\mathrm{x} 4.0 \mathrm{~cm}$ mass having central air locule. Mass was located medial to the left lobe of liver along lesser curvature of stomach (Figure 3). Colon appeared normal on CT scan. UGIE showed large smooth surface polypoidal mass in the proximal body of stomach. Sleeve resection of gastric mass was performed. The colon was normal during surgery.

The tumor grossly measured $4.5 \times 4.0 \times 2.5 \mathrm{~cm}$ and was localized within the gastric wall. The cut surface of tumor showed solitary fleshy lesion with whorled pattern (Figure 4). Microscopically, mucosa of stomach was intact with tumor localized within the sub-mucosa extending into the muscularis propria. Tumor had spindle cells in fascicles with dense infiltration of lymphocytes and peripheral lymphoid 


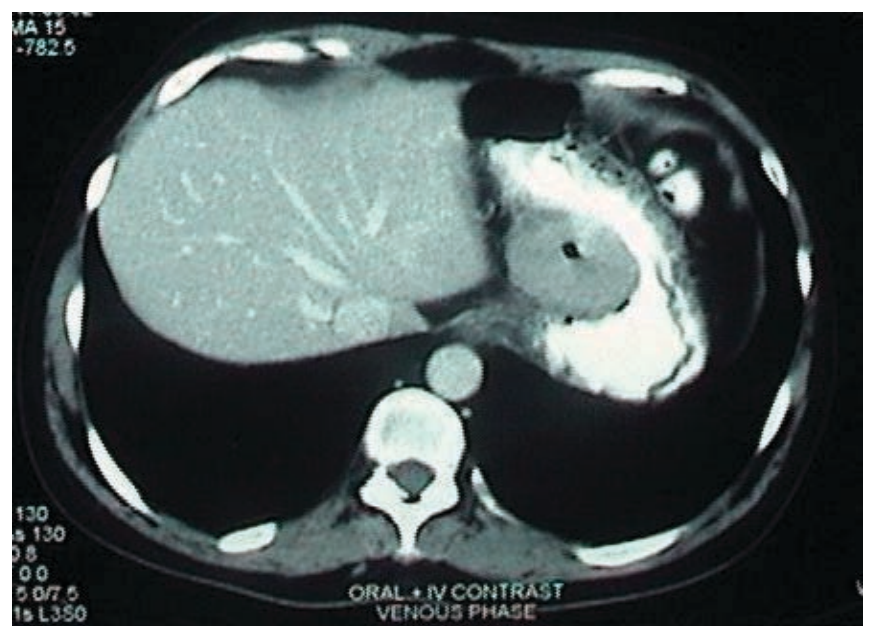

Figure 3: Hypodense lesion in body of stomach.

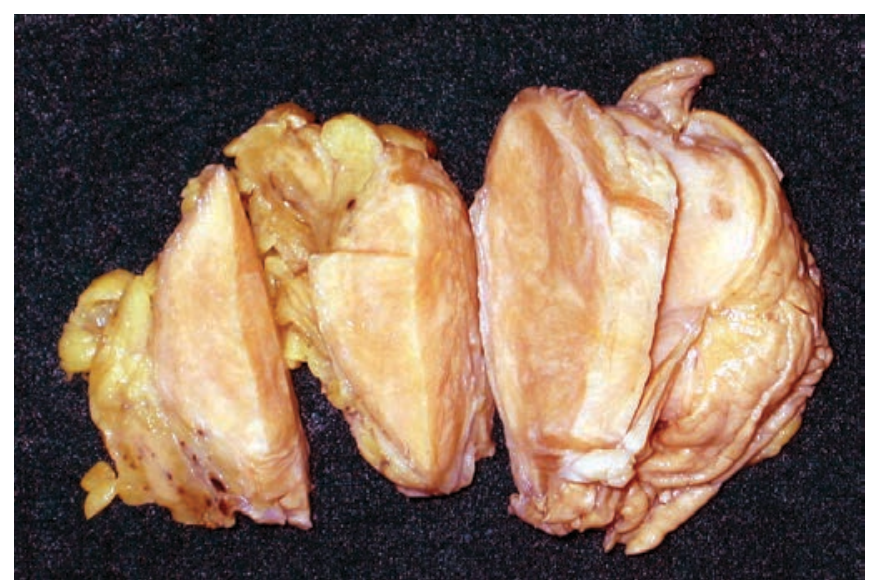

Figure 4: Gastric sleeve resection specimen (4.5 X 4 X $2.5 \mathrm{cms})$ showing a solitary fleshy glistening lesion.

cuffing (Figure 5). Mitotic figures were 2/50 high power field. Stroma was myxoid with focal hyalinization having areas of necrosis. IHC of tumor cells was positive for S-100 protein (Figure 6), and negative for CD 117, CD 34, DOG1 and smooth muscle actin (SMA), confirming gastric schwannoma Ki67 index was 1\%.

All three patients are doing well at 5, 8 and 12 months of follow up respectively. Endoscopy and ultrasound abdomen did not show any recurrence.

\section{Discussion}

Gastrointestinal schwannoma is a rare and distinctive gastrointestinal mesenchymal tumor. They were first described by Daimaru et al in $1988 .{ }^{1}$ Less than 50 cases have been reported in literature so far. ${ }^{2}$ The cell of origin of Schwannoma is Schwann cell of the enteric nervous system as confirmed on electron microscopy. ${ }^{3}$ They are also known as neurinomas or neurilemmomas. Gastrointestinal schwannomas are rare and distinctly different neoplasms from conventional schwanomas that arise in soft tissue or the central nervous system.

The clinical presentations are usually non-specific, and include epigastric discomfort, dyspepsia, or abdominal pain. Most of the cases are anymptomatic for a long period of time. One of our cases presented with upper gastro-intestinal

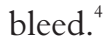

These tumors are more often seen in middle and late adult age, with a peak in sixth decade of life. There are few observations of female predilection. In our present case series two were female.

Endoscopic evaluations are usually nonspecific as these tumors appears grossly as sub mucosal or exophytic tumors with or without a central ulceration. UGIE in all three cases had submucosal polypoidal mass.

Imaging findings are helpful in explaining the cause of symptoms as well as to determine the best approach to treatment. Endoscopic sonography (EUS) has been considered as the best diagnostic modality. EUS can assess the size and origin of gastric submucosal tumor and define the

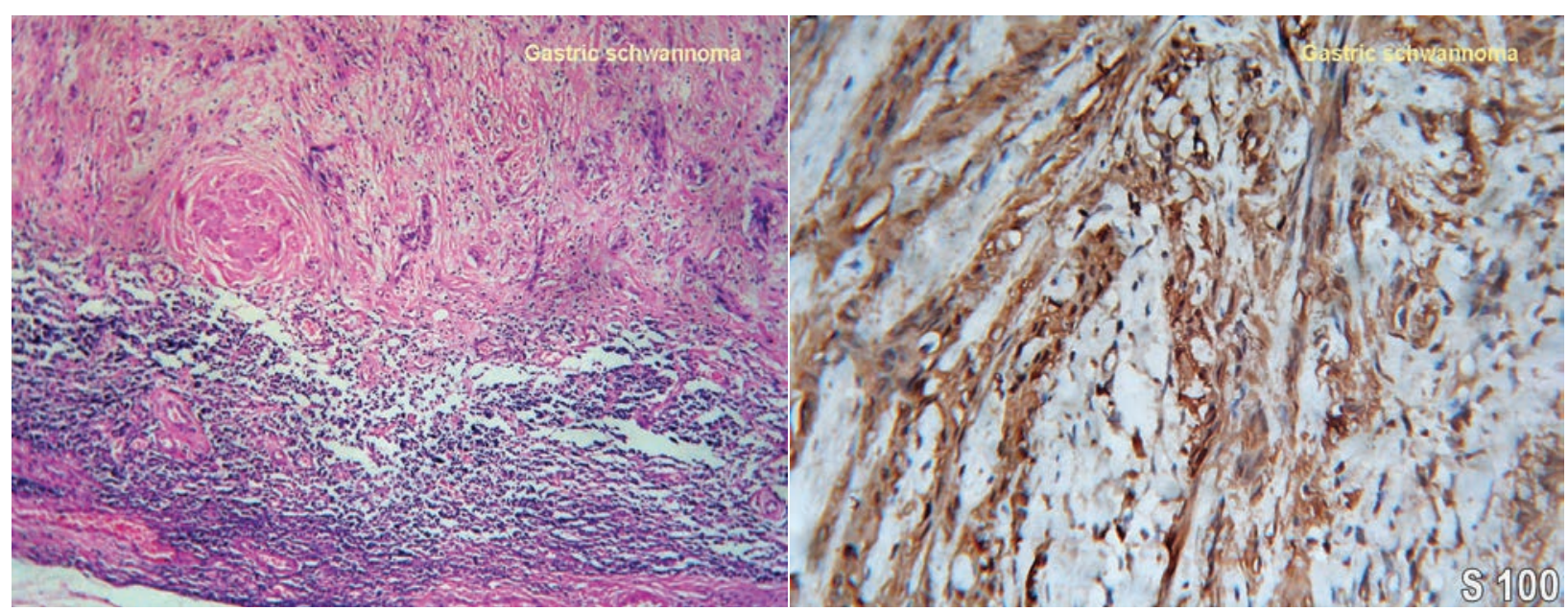

Figure 5: H \& E - 100X - Spindle cells in fascicles with peripheral lymphoid cuffing
Figure 6: IHC - S100 marker - 400X - Tumor cells are positive for $\mathrm{S} 100$ marker 
location and extent of tumor with displacement of surrounding organs or vessels. They are no definite diagnostic criteria for diagnosis of schwannoma through trans abdominal sonography which shows as a homogeneously hypoechoic mass. Two of our cases had gastric wall mass on ultrasound. Schwannomas on computed homography are homogeneously attenuating, well defined mural masses. MRI demonstrates a discretely marginated mass with well defined lobulations and absence of necrosis. CT scan and MRI are mainly useful in the detection and characterization of large gastric submucosal or exphytic tumor and its relation with surrounding organs. ${ }^{5}$

These tumors are usually benign and slow growing. When tumor presents as an exophytic polypoidal growth arising from the gastric wall to the abdominal cavity, the differentiation of schwannoma from other submucosal tumors is difficult on pre-operative examination alone. Other gastrointestinal mesenchymal tumors comprising of leiomyomas, leiomyosarcomas, and gastrointestinal stromal tumors (GIST) form the main differential diagnosis. ${ }^{6}$

The diagnosis of schwannoma is based on tissue histology with further confirmation by immunohistochemical markers. ${ }^{7}$ Schwannoma of GI tract are rare. Most common site along the GI tract is the stomach. Tumors arising from colon and esophagus have also been reported. ${ }^{8}$ Gastric schwannomas represent only $0.2 \%$ of all gastric tumors and $4 \%$ of all benign gastric tumors. ${ }^{9}$ Malignant transformation of a gastric schwannoma is very rare. Majority are submucosal in origin, located in body of stomach with size ranging from 0.5 to $11 \mathrm{~cm}$ in earlier reported cases. Two of our cases were located in the body and one in gastric antrum, and size varied from 3-5 cm. Gastric schwannomas are not associated with multiple neurofibromatosis.

Microscopically these tumors are homogeneous, rubbery to firm. Cut surface is whitish yellow and glistening. They are usually solitary or multilobulated lesions with or without necrosis and hemorrhage. In our case series, all tumors were solitary, well defined, with two cases showing small areas of necrosis and myxoid change.

Histologically, tumors are reported to be well circumscribed, but unencapsulated. Schwannomas arise in the submucosa and muscularis propria.

They are composed of interlacing bundles of spindle cells arranged in whorls, storiform pattern or sheets. Nucleus is spindle shaped with occasional wavy nuclei. Verocay bodies may be present. Mitosis is few which was also observed in our series. Interspersed are inflammatory cells primarily mature plasma cells, lymphocytes and histiocytes. Myxoid areas and mild vascularised stroma are noted.

The characteristic feature of gastrointestinal tract schwannoma is peripheral infiltrate of mature small lymphocytes with a peripheral cuff of lymphoid aggregates around the tumor which helps in differentiating from GIST. ${ }^{10}$ This distinctive feature was also noted in all three cases.

Immuno-histochemistry shows strong nuclear and cytoplasmic staining pattern for S100 protein, vimentin and glial fibrillary acidic protein (GFAP) with consistent negativity for CD117, DOG1, CD34 and Smooth muscle actin S-100 is a schwannian cytosolic calcium binding protein. S-100 protein positivity enables differentiation from other tumors of smooth muscle origin which may have overlapping histological and cytological features, the most

Table 1: Comparison of smooth muscle tumors of gastrointestinal tract

\begin{tabular}{|c|c|c|c|c|}
\hline SI.No & Features & Schwannoma Mural Mass & GIST(Gastrointestinal Stromal Tumors) & Leiomyoma of Gastrointestinal tract \\
\hline 1. & Gross & $\begin{array}{l}\text { Rubbery to firm, Yellow - } \\
\text { white to tan, glistening } \\
\text { and often trabeculated } \\
\text { cut surface }\end{array}$ & $\begin{array}{l}\text { Mural mass firm to soft or fish - } \\
\text { fleshy, tan, gray pink or variegated } \\
\text { tumor with degenerative changes. }\end{array}$ & Mural mass, gray white \\
\hline 2 & Histology & $\begin{array}{l}\text { Moderately cellular } \\
\text { Nuclear pleomorphism(minimal) } \\
\text { Rare MF } \\
\text { Characteristic feature } \\
\text { peripheral lymphoid cuff }\end{array}$ & $\begin{array}{l}\text { Highly cellular (Spindle cell or epithelioid) } \\
\text { pleomorphism }+/- \\
\text { MF }+/- \\
\text { Not seen }\end{array}$ & $\begin{array}{l}\text { Paucicellular, Spindle Cells } \\
\text { Minimal nuclear pleomorphism } \\
\text { No lymphoid cuff }\end{array}$ \\
\hline 3 & $\mathrm{IHC}$ & $\begin{array}{l}\text { S- } 100+v e \\
\text { GFAP + ve } \\
\text { CD } 117-v e \\
\text { CD } 34-v e \\
\text { Desmin -ve } \\
\text { SMA -ve } \\
\text { DOG-1-ve }\end{array}$ & $\begin{array}{l}\text {-ve } \\
\text {-ve } \\
\text { +ve } \\
\text { +/- } \\
\text { Variable } \\
\text { Variable } \\
\text { +ve }\end{array}$ & $\begin{array}{l}-v e \\
-v e \\
-v e \\
-v e \\
+v e \\
+v e \\
-v e\end{array}$ \\
\hline 4 & $\begin{array}{l}\text { Cell of } \\
\text { origin }\end{array}$ & Schwann cell & titial cell of cajal & Smooth muscle \\
\hline
\end{tabular}


common differential diagnosis being GIST (Table 1). All three cases of our series were positive for S 100 marker and negative for CD 117, DOG1, CD 34 and SMA markers.

Gastrointestinal stromal tumors, particularly gastrointestinal autonomic nerve tumors (GANT subtype) require exclusion. GANT tumors are CD117, DOG1 and CD34 positive and negative for S-100 protein. GANT tumor cells do not have basement membranes but have cytoplasmic dense core granules on electron microscopy. Gastric schwannoma are cured by local resection - surgically or wherever possible through endoscopic means ${ }^{11}$ the prognosis after tumor resection in excellent.

In conclusion, gastric schwannoma represent a distinct uncommon benign tumor which is to be differentiated from other gastric mesenchymal tumors. A definitive diagnosis is important because the treatment of other mesenchymal tumors may differ. Schwannomas can be surgically or if possible, endoscopically resected. They carry good prognosis when compared to the more aggressive gastro-intestinal stromal tumors which have malignant potential.

\section{References}

1. Daimaru Y, Kido H, Hashimoto H, Enjoji M. Benign Schwannoma of the gastrointestinal tract: a clinicopathologic and immuno-histochemical study. Hum Pathol 1998;19:257-64.

2. Carlos A Iwamolu, Carlos F Garcia, Maysoon Razzak. A 23year-old woman with a polypoid gastric mass. Archives of Pathology and Laboratory Medicine 2003;127:43 - 44.

3. Steven Latosinsky. Case report Gastric Schwannoma: a rare Schwann cell tumour of the GI tract. UWOMJ. Summer
2011; 80: S1.

4. Mam Chandra, Punit Mehrotra, Manoj Kumar Mitra. Gastric schwannoma presenting as gastric polyp with gastrointestinal bleeding. Indian J Gastroenterol 2002;:21:31.

5. Menno H Raber, Cathelijne MP, Ziedses des Plantes, Robert Vink, Joost M Klaasea. Gastric Schwannoma presenting as an incidentaloma on CT-Scan and MRI. Gastroenterology Research 2010;3:276-280.

6. Lee JR, Joshi V, Griffin JW Jr, Lasota J, Miettinen M. Gastrointestinal autonomic nerve tumor: immunohistochemical and molecular identity with gastrointestinal stromal tumor. Am J Surg pathol 2001; 25:979-987.

7. Prévot S, Bienvenu L, Vaillant JC, de Saint-Maur PP. Benign schwannoma of digestive tract: A clinco-pathologic and immuno-histochemical study of five cases, including a case of esophageal tumor. American Journal of Surgical Pathology 1999; 23:431-36

8. Miettinen M, Shekitka KM, Sobin LH. Schwannomas in the colon and rectum: a clinico-pathologic and immunohistochemical study of 20 cases. Am J Surg Pathol 2001;25: 846-55.

9. Lin CS, Hsu HS, Tsai CH, Li WY, Huang MH. Gastric Schwannoma. JChin Med Assoc 2004;67:583-6.

10. Sarlomo-Rikala M, Miettinen M. Gastric schwannoma - a clinico-pathological analysis of six cases. Histopathology 1995; 27:355-60.

11. Silecchia G, Materia A, Fantini A, Spaziani E, Picconi T, Trentino P, Faticanti Scucchi L, Basso N. Laparoscopic resection of solitary gastric schwannoma.J Laparoendosc Adv Surg Tech A 1997;7:257-63.

Source of support: Nil; Conflict of interest: none declared 